\title{
Using 3D Scanning for Accurate Estimation of Termination Points for Dimensional Quality Assurance in Pipe Spool Fabrication
}

\author{
Mohammad Mahdi SHARIF', Christopher RAUSCH${ }^{2}$, Sidy NDIONGUE ${ }^{3}$, Carl HAAS ${ }^{4}$, Scott WALBRIDGE $^{5}$ \\ ${ }^{1}$ PhD Candidate, Department of Civil and Environmental Engineering, University of Waterloo, Canada \\ ${ }^{2}$ PhD, Department of Civil and Environmental Engineering, University of Waterloo, Canada \\ ${ }^{3}$ BSC, Department of Electrical Engineering, University of Waterloo, Canada \\ ${ }^{4}$ Professor, Department of Civil and Environmental Engineering, University of Waterloo, Canada \\ ${ }^{5}$ Professor, Department of Civil and Environmental Engineering, University of Waterloo, Canada
}

Submitted May 15, 2021

Accepted September 8, 2021

Published October 2021

\section{Corresponding Author}

Mohammad Mahdi Sharif PhD Candidate

m33shari@uwaterloo.ca

Department of Civil and Environmental Engineering University of Waterloo 200 University Avenue West Waterloo, ON, Canada N2L 3G1

DOI http://doi.org/10.29173/ijic253

Pages 54-69

ISSN 2563-5034

\section{ABSTRACT}

Increased prefabrication and modularization have resulted in fabrication shops producing more complex assemblies with tighter tolerances. Most measurements in fabrication shops are still done using manual tools that are not accurate enough for engineering tolerance specifications, which can lead to rework. Three dimensional (3D) scanning and measurement systems can provide increased accuracy and digital integration capabilities, however they do not sufficiently support fast and accurate dimensional quality assurance (DQA) of pipe spool fabrication. This is because no dimensional quality assurance methods to date have focused solely on termination points for pipe spool assemblies. In the present article, a new scan-vs-BIM method is developed to accurately estimate termination points for 3D scanned cylindrical assemblies. This method relies on statistically fitting circular features at termination points and thus eliminating conventional issues with target placement for laser trackers and measurement readings for tape measures. The method is tested in an industrial-scale experiment, where 30 pipe spool assemblies were fabricated, and more than 400 quality control steps completed. The accuracy of termination point detection was benchmarked against results from a laser tracker and compared against commercial scan-to-BIM software. Results show that the developed method has an average accuracy of $1.01 \mathrm{~mm}$ and is significantly better than the scan-to-BIM software with an average accuracy of $4.75 \mathrm{~mm}$.

\section{KEYWORDS}

Prefabrication; 3D laser scanning; Dimensional quality assurance 


\section{Introduction}

The increased adoption of modularization and prefabrication has allowed for enhanced quality of the final built product. While these trends also allow for automated and controlled processes to increase quality, safety, and productivity [1-4], the continued existence of onsite rework remains a significant cost and schedule impediment for these types of projects [5]. This is especially true in complex pipe spool fabrication projects, where the geometry of assemblies is typically bespoke and intricate. Depending on the type of project and enumeration approach, rework can amount to $3 \%$ to $10 \%$ of a project's cost [6,7]. Rework manifests itself in a variety of forms in piping projects, and only some of it is documented by project participants. Examples of costly rework scenarios that can be avoided by improved measurement and data communication tools include modules not mating due to error in one or more termination points onsite, bolt-hole misalignment in flange connections, re-cutting and re-welding of assemblies due to design misinterpretation, delayed approval and shipment of assemblies due to QC (quality control) processes becoming a bottleneck, fabrication worker confusion due to design changes, and repetitive measurement due to tight tolerances and lack of utilization of fit-for-purpose tools. In general, the use of Dimensional Quality Assurance (DQA) tools has the primary objective to increase and maintain an adequate level of quality. As a by-product, DQA tools also have the potential to save project costs by avoiding rework, material waste, and increasing productivity. Conventional measurement tool kits can be divided into two groups: (1) manual hand measurement tools such as tape measures, bubble levels, and straight edges, and (2) advanced surveying grade tools such as total stations and laser trackers. To perform the required quality control measurements, both of these methods are similar in their use of termination points. Termination points are identifiable parametric features on assemblies that are idealized by points. The distance and angle between termination points on assemblies are calculated and compared with the allowable tolerance value. While they are accurate, surveying-grade tools are seldom used in fabrication shops due to their complexity and high cost of operation. As such, fabrication workers use manual measurement tools for most of their measurements. The lack of an advanced, accurate, and integrated measurement approach in fabrication shops is one of the main root causes of geometric rework incidents [8].

\subsection{Research Scope and Problem Definition}

This research focuses on improving the dimensional quality of piping element fabrication, since this can amount to $50 \%$ of the total cost of an industrial construction project [9]. The range of materials used, coupled with the extensive hands-on time from craft workers, makes the piping portion of projects costly and time-consuming. Furthermore, incorporating automated dimensional quality assurance processes into the piping industry is challenging yet necessary, because most components in a piping project are bespoke. Most measurement is still conducted using manual hand measurement tools such as tape measures, bubble levels, squares, and straight edges.

This research proposes a new measurement method that utilizes 3D scan data and can deliver rapid dimensional quality control data based on termination points. The utilization of 3D scan data is advantageous over existing tools, since 3D scan data can provide superior visualization and can be integrated as part of the fabrication process, as shown by [3]. The delivery of information based on termination points is critical for two reasons: (1) the abstraction of termination points allows for the development of a computationally efficient and accurate approach, and (2) the utilization of termination points in delivering the quality control information would allow for seamless integration into fabrication shops' processes without extensive training or process changes, since existing QC frameworks rely on measurement and communication of tolerances using termination points.

The challenges being addressed in this research are that existing methods for DQA of pipe spool fabrication are often too time-consuming for efficient deployment, provide non-structured or inadequately abstracted information, and often sacrifice accuracy at termination points to achieve overall minimum average error reduction. These challenges are overcome using a new framework and algorithms that efficiently provide accurate information of termination points. While the level of required accuracy remains high for pipe spool DQA, as the cost of 3D scanners continues to decrease, efficient processing of point cloud data will have a significant role in complex infrastructure projects.

\section{Dimensional Quality Assurance for Pipe Spool Fabrication using 3D Point Clouds}

The use of 3D point clouds for dimensional quality assurance (DQA) of pipe spool fabrication has existed in a range of research projects for well over the past decade. While other digitization approaches have also been explored, such as 2D image analysis, they often have fundamental challenges for inspection of pipe spool assemblies within a fabrication facility such as featureless pipe surfaces, lighting issues from weld flashes, and the dynamic setup requirements for complex pipe spools which cannot guarantee consistent camera pose between setups $[10,11]$. An example of infrastructure projects with complex pipe spool fabrication process is the refurbishment of existing nuclear reactors. Due to the high accuracy requirements for these types of infrastructure projects, laser scanners are uniquely positioned among other types of digitization devices for conducting DQA. The ranging error of laser scanners can 
be as low as $1 \mathrm{~mm}$ (ranging error for the scanner used in this study is defined as a systematic measurement error at around $10 \mathrm{~m}$ and $25 \mathrm{~m}$ ) and have the ability for rapid data collection.

Researchers have adopted the use of 3D point cloud approaches to circumvent many of the conventional challenges associated with other digitization approaches. In general, there are two main ways to instantiate such DQA processes: with or without an existing as-designed 3D model [12]. Such approaches are summarized in Figure 1 and reviewed in detail in the subsequent sections.

\subsection{Dimensional quality assurance without a $3 D$ design model}

In many instances, fabrication shops do not have access to the $3 \mathrm{D}$ design model of the assemblies they need to build. This happens because of multiple reasons including: (1) interoperability issues between various software, (2) lack of contractual obligations to transfer the 3D model, (3) 3D models usually correspond to engineering ISO drawings not fabrication shop drawings. As such, Scan-to-BIM methods are used in these instances. When an existing 3D design model of the pipe spool being fabricated is not available, feature detection algorithms can be used to extract curvature-based features in a point cloud and compared with information contained in 2D drawings. These methods can be categorized under prevailing work described as "scan-to-BIM", where a 3D model is re-created from a point cloud. Previously, Ahmed et al. [13] used Hough Transform on cross- sectional slices of pipe assemblies to extract pipe radii of known sizes. A shortcoming of the method described by Ahmed is that it assumes the orientation of the pipe spools is parallel or perpendicular to the scanner's Z-axis. Son et al. [14] used curvature feature extraction from local surface patches at known locations prescribed on piping and instrumentation diagrams (P\&IDs) and subsequently fit non-uniform rational basis spline (NURBS) geometry to generate pipe spool model elements. Wang et al. [15] used Density-Based Spatial Clustering of Applications with Noise (DBScan) to help generate complete pipe spool assembly models from 3D point clouds. While these algorithms may rely on a set of pre-existing parametric objects (oftentimes of set sizes) to fit to a segmented point cloud, other methods have also been developed which reconstruct model elements using more granular geometric techniques to minimize discrepancies with the as-built conditions. For instance, Dimitrov et al. [16] developed a generalizable technique that fits NURBS geometry to constructed elements that do not need to conform to strict parametric assumptions (e.g., a pipe must be perfectly straight). The significance of techniques like this is that they avoid the errors accrued by trying to best-fit straight pipe elements to as-built features which may have non-negligible deformation such as from welding distortion. However, the downside of these approaches is that inferring discrete errors between asbuilt and as-designed states (represented by drawings) is hard, and relies on tedious, timely analyses.

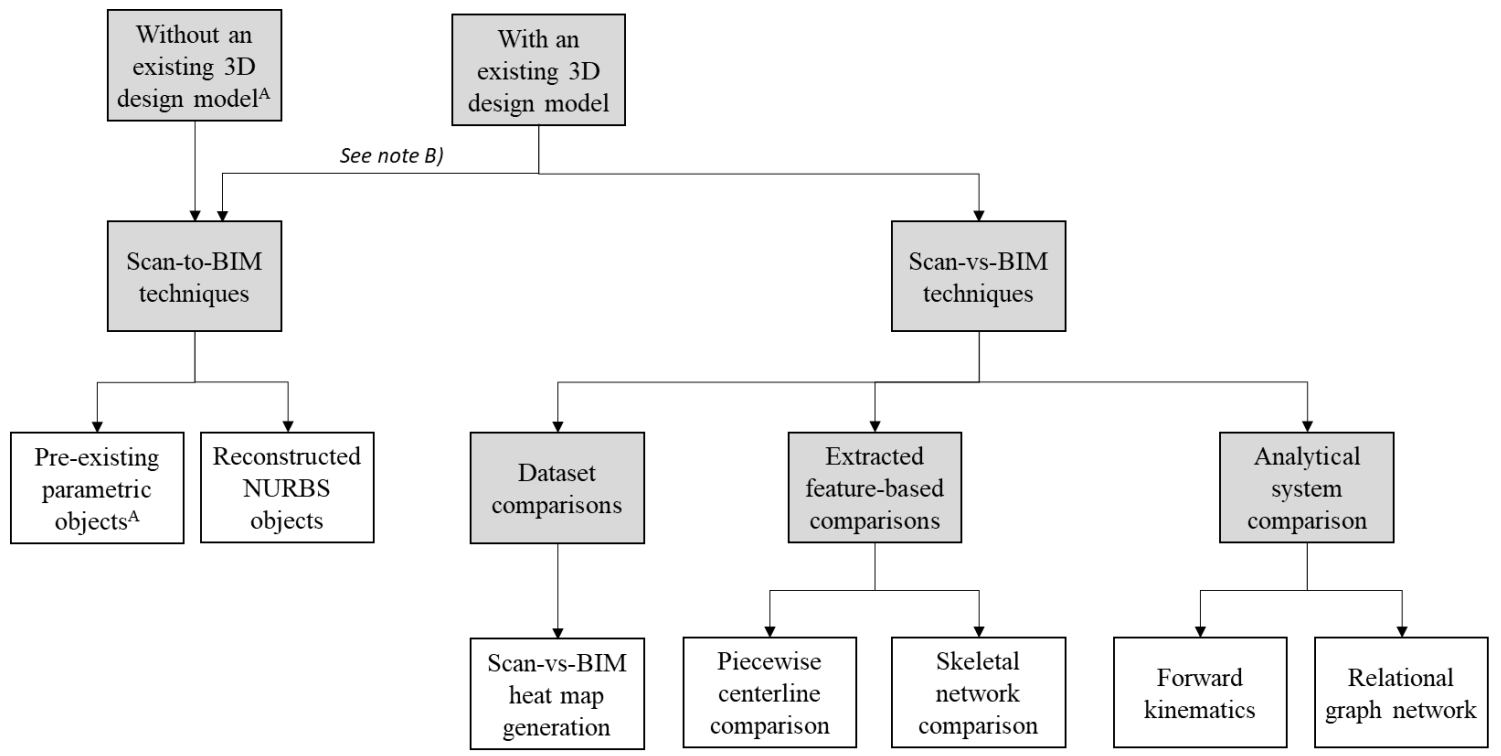

A) "3D design model" refers to the distinct model of the pipe spool assembly being fabricated, whereas "pre-existing parametric objects" refers to a library of potential parametric pipe spool objects

B) A 3D model produced through Scan-to-BIM can be compared with as-designed BIM for DQA. Such comparison is likely to be indirect (e.g., comparing the termination points of an assembly from the as-designed $3 D$ model with those produced from the scan-to-BIM model).

Figure 1. Overview of existing methods for dimensional quality assurance for prefabricated pipe spool assemblies using 3D point cloud data 
Across existing scan-to-BIM methods for pipe spool fabrication, perhaps the most useful (and recent) technique has been developed by Maalek et al. [17]. Their method extracts the center and orientation of key pipe flanges on assemblies using algorithms such as the Pratt's circle fitting algorithm and the squared Mahalanobis distance computation. While the most conducive to pipe spool fabrication DQA, this method still requires a subsequent comparison of as-built features (i.e., flange centers and plane orientations) with as-designed information, which can be tedious and time-consuming. Additionally, many commercially available software packages rely on human operators to manually find objects and to best-fit parametric objects into point clouds. In their recent work, Essnashary et al. [18] have investigated the resulting fundamental imprecisions when human operators are involved in scan-to-BIM methods.

\subsection{Dimensional Quality Assurance with a design model} While notable works have emerged to help conduct DQA when an existing model is not available or developed to a suitable level of detail, far more suitable methods have been developed for directly comparing 3D point clouds with existing as-designed models (i.e., "scan-vs-BIM"). Not only does this approach circumvent extensive subsequent evaluations to understand and abstract information for DQA, but these processes do not strictly require a semantically rich BIM for engendering full automation. Often, a "semantically sparse" CAD model (e.g., stereolithography format) is suitable, as this paper later posits and defends. Upon surveying existing methods for DQA within scan-vs-BIM, there are three distinct sub-categories: (1) direct dataset comparisons, (2) extracted feature-based comparisons, and (3) analytical system comparisons.

\subsubsection{Direct dataset comparisons}

The most basic, yet perhaps pervasive scan-vs-BIM method is directly overlaying a scan on a model and depicting deviations using a heat-map. This method can be used to visualize overall Euclidean distance-based deviations between an as-built and as-designed state. However, the challenge of using this method for DQA in pipe spool fabrication is that not all deviations can be directly inferred by Euclidean distances, for instance, if a pipe is shifted along its principal axis, deviations may not be appropriately displayed (in contrast to a case where a pipe is rotated about that same axis, whereby a linear propagation in a heat-map would be displayed). Given the limitations of direct heat-map comparison, researchers have adopted more advanced methods such as the use of random sample consensus (RANSAC) to extract pipe segments and perform deviation analysis using orientation comparisons of pipes [19].

\subsubsection{Extracted feature-based comparisons}

Since direct dataset comparisons do not capture all of the types of discrepancies that can exist for pipe spool fabrication, researchers have used extracted feature-based comparisons for improved DQA. The most basic form of this involves extracting the centreline of pipes (including start and end nodes) and comparing this data with similar features from an as-designed model. Since the model does not need to be semantically rich, this method is efficient and robust. In their work, Guo et al. [20] use this form of centerline comparison after extracting straight pipe segments in MEP modules. While their method achieves a suitable accuracy $(3.78 \mathrm{~mm})$, it cannot be directly used for more complex assemblies which have various angles and joints. This is where other techniques such as comparing skeleton networks as posited by Nahangi et al. [21] may be more suitable. In this method, the point cloud is converted into a skeletal model by extracting crosssections of objects and fitting lines through the centre of each cross-section. An input BIM is also used to instantiate the skeletal candidates (i.e., radius of pipe at key locations), and to infer the deviation of the as-built status to the design intent. This is then carried out for an entire pipe assembly. In general, the use of extracted feature-based comparison may be useful for understanding potential realignment measures, these methods involve more computation than strictly required for an initial DQA assessment of termination points on pipe spool assemblies. Furthermore, occlusions in point cloud data lead to challenges when generating centerlines through an assembly.

\subsubsection{Analytical system comparison}

A final way to utilize an as-designed model for DQA in pipe spool fabrication is the use of advanced analytical system comparisons. Such techniques are related to extracted features yet assume or represent pipe spools as analogous analytical systems. In their work, Nahangi et al. [22] use the analogy of kinematic chains to identify errors and posit realignment measures. First, forward kinematics is used to compute the discrepancy between as-built and as-planned pipe spool segments. Such computation relies on the assumption that pipes can be modelled and behave similarly to joints in robotics systems (i.e., rotations and translations about joints). This method, while powerful for potentially providing near real-time feedback on how to correct defective assemblies, relies on having sufficient point cloud coverage of pipe spools. Furthermore, the results of this method are currently not conducive for quick termination point checking (i.e., centre points and alignment of pipe flanges). Other analytical systems comparisons have involved the use of graph theory to abstract and track the accumulation of error in pipes. This technique, as outlined by Kalasapudi et al. [23], requires establishing a comprehensive tolerance network associated with each pipe element and subsequently quantifying and comparing the errors of each associated pipe element. In summary, while several innovative techniques can be used to abstract errors in pipe spool assemblies, 
oftentimes, these approaches are far too comprehensive to adopt for real-time DQA of termination points.

\subsection{Knowledge gap and research contribution}

While existing methods for DQA of pipe spool fabrication using 3D point clouds have several valueadding capabilities, in general, they have the following limitations which this paper aims to address:

- Primitive fitting techniques often sacrifice accuracy at termination points in order to achieve an overall minimum average error reduction.

- Pipe spool termination features are often regularly shaped primitives, which do not require the same level of complexity or sophistication to process and analyze as irregularly shaped primitives - whereby potentially more efficient algorithms can be used to decrease computational cost/time.

- No methods to date have delivered approaches for efficient DQA of termination points on pipe spool assemblies, which continues to be an essential (yet rudimentary) requirement for prefabricated project execution.

Finally, while a range of devices can be used to generate a 3D point cloud (such as projector-camera systems, photogrammetry, range cameras, laser vision sensors, etc.) $[10,24,25]$, the use of 3D laser scanners is targeted in this work, given their widespread adoption and proven reliability in industry for high accuracy, speed, insensitivity to lighting conditions, range and high density - all of which are highly conducive to pipe fabrication in offsite facilities as demonstrated by Guo et al. [20].

\section{Methodology}

The methodology section of the present study is broken into two main sections. Initially, the required definitions and classification of termination points are provided. Secondly, the method for developing a termination pointbased scan-vs-BIM method is explained. The method was used as part of the fabrication of 30 pipe spools. The results of using the method are explained in Section 4.

\subsection{Termination Point Definition and Classes}

We define termination points as local coordinate systems where assemblies are connected or constrained.

Furthermore, termination points are identifiable parametric features on assemblies that are idealized by points. The detection of termination points is part of the fabrication process. For example, the center point of flanges is often used as a termination point. As seen in Figure 2, a fabrication worker is using manual measurement tools to draw the centerlines on the flanges of a pressure vessel to allow subsequent quality control measurements.

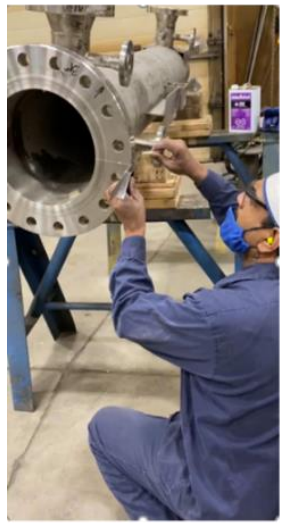

(a)

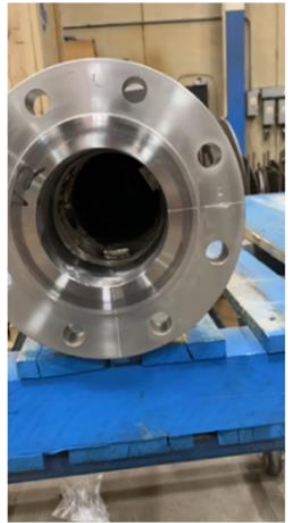

(b)

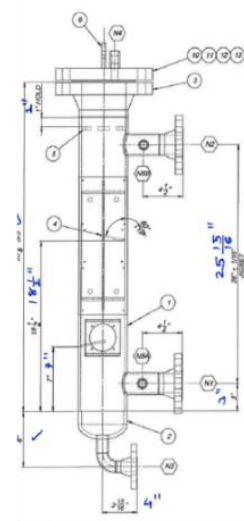

(c)
Figure 2. The use of termination points as part of the fabrication process. (a) A fabrication worker using manual measurement tools to detect the centerline on a flange. (b) the centerline marked on the flange using chalk. (c) the required QC measurements using termination points

To allow for the development of a comprehensive framework, researchers have defined a new framework for the classification of termination points on structures that would allow building new applications and tools in a way that is generalized [26]. The 10 classes of termination points are as shown in Tables 1 and 2.

Table 1. Termination point classes in the 3D-model and As-built spaces

\begin{tabular}{lcc}
\hline Termination Point Class & 3D-model & As-built \\
\hline (A): Origin termination point & $A_{m}$ & $A_{s}$ \\
(B): Destination termination & $B_{m}$ & $B_{s}$ \\
point & & \\
(C): Assembly mate-to-origin & $C_{m}$ & $C_{s}$ \\
termination point & & $D_{s}$ \\
$\begin{array}{l}\text { (D): Assembly mate-to- } \\
\text { destination termination point }\end{array}$ & $D_{m}$ & \\
(E): Assembly non-mate & $E_{m}$ & $E_{s}$ \\
termination point &
\end{tabular}

Table 2. Termination vector classes in the 3D-model and As-built spaces

\begin{tabular}{lcc}
\hline Termination Vector Class & 3D-model & As-built \\
\hline (F): Normal-to-origin & $\overrightarrow{F_{m}}$ & $\overrightarrow{F_{s}}$ \\
termination plane & $\overrightarrow{G_{m}}$ & $\overrightarrow{G_{s}}$ \\
(G): Normal-to-destination & $\overrightarrow{H_{m}}$ & $\overrightarrow{H_{s}}$ \\
termination plane & $\overrightarrow{I_{m}}$ & $\overrightarrow{I_{s}}$ \\
(H): Normal-to-assembly & $\overrightarrow{J_{m}}$ & $\overrightarrow{J_{s}}$ \\
mate-to-origin plane & &
\end{tabular}


Table 1 provides the definition for termination point coordinates in the 3D model space (subscripted by $\mathrm{m}$ ) and 3D scan space (subscripted by s). Table 2 has definitions related to the orientation of termination points in the scan and the 3D model spaces. The definitions of the classes in Table 1 and Table 2 are as follows:

- Class A: The point to which the installation process is first attempted.

- Class B: The point(s) to which the installation process is attempted once the origin termination point has been locked into place.

- Class C: The point to which the receiving assembly (or site location) is mating with the origin termination point.

- Class D: The point to which the receiving assembly (or site location) is mating with the destination termination point.

- Class E: A point that is not mating with another assembly but is still important to measure from or to.

- Class F: The orientation of the origin termination point.

- Class G: The orientation of the destination termination point(s).

- Class H: Orientation of the mating location of the receiving assembly or site location to the origin termination point.

- Class I: Orientation of the mating location of the receiving assembly or site location to the destination termination point(s).

- Class J: Orientation of the none mate termination point(s).

\subsection{Termination Point-Based Scan-vs-BIM for Pipe Spool Assemblies}

In the described method in this section, fabrication errors are visualized by providing a comparison between a 3Dscanned point cloud of an as-built assembly and its corresponding 3D-model (as-designed) point cloud. The described method uses termination points to provide the overlay comparison between the scanned point cloud and the model point cloud. Utilization of the detected termination points in providing the comparison is critical for fabrication dimensional quality assurance processes because the current quality control requirements dictate the measurements from and to termination points. Additionally, the tolerance requirements on termination points are often tighter than other fabrication tolerances (since they are the mating locations between different assemblies, and because of the rigidity of the assemblies the interdependence geometrically between multiple simultaneous termination points can be complex), and fabrication workers need exact geometric data for these points. The required steps for the termination-point-based overlay comparison are summarized in Algorithm 1. C\# programming language was chosen to implement the proposed algorithm.

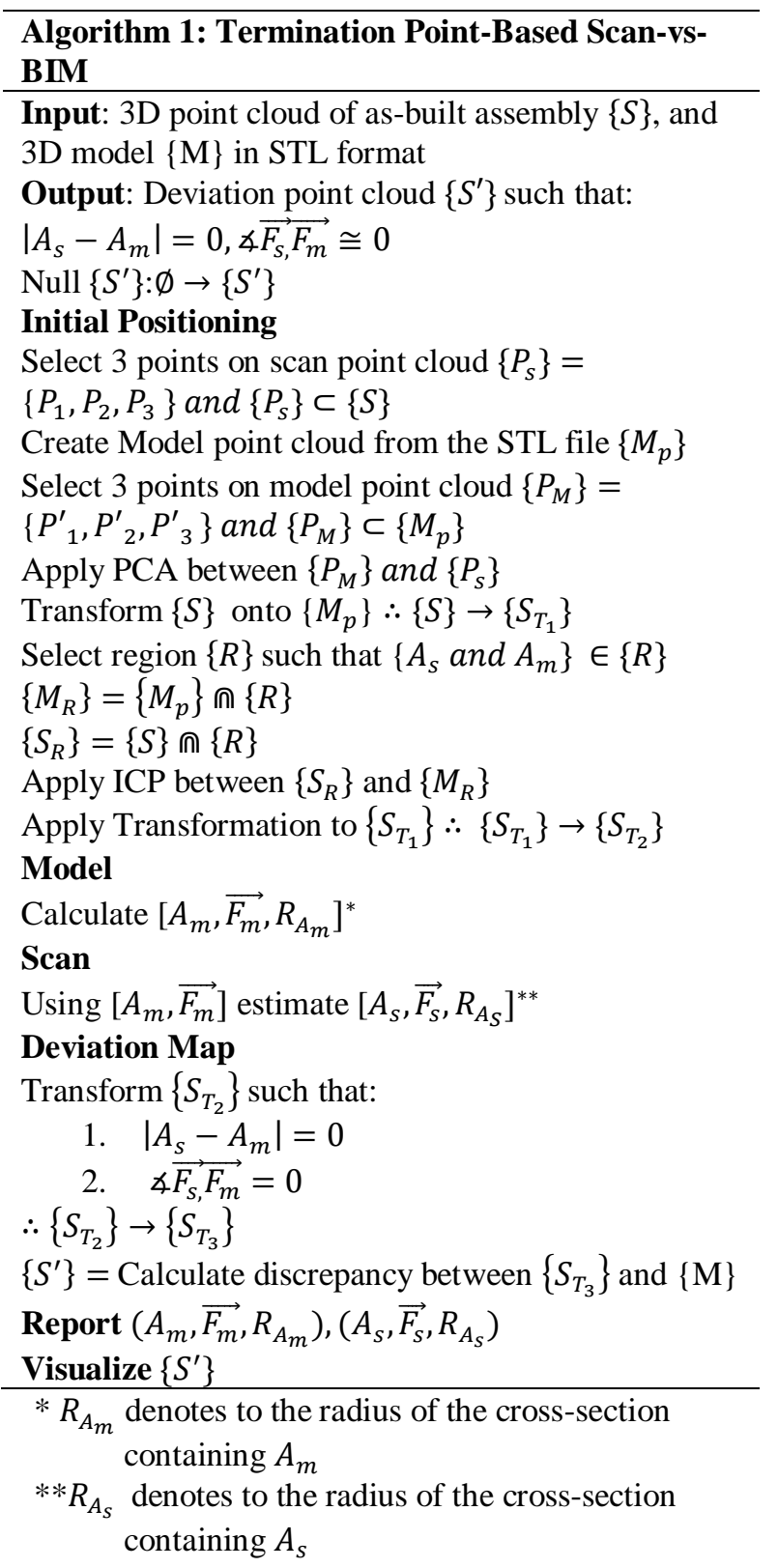

\subsubsection{STereoLithography format (STL)}

Many design firms consider their specific CAD formats proprietary and refrain from sharing them with the fabricators. To accommodate this limitation and to develop a generalized method, this study relies on an STL format of the model. This format stores the model data by tessellating the surfaces in triangles and storing the normal vector for each triangle (it has no other property and thus, design firms are more willing to share this data type). The definition of a mesh is provided in the next section. Figure 3 shows the difference in various model representations.

3.2.2 Generation of 3D model point cloud from the mesh In order to create the point cloud of the 3D model, the first step is to convert the CAD design (where the assembly is defined parametrically) into a mesh object. A mesh is 
defined as a collection of vertices, triangles, quadrilaterals (quads), or other simple convex polygons (n-gons) that define the shape of a polyhedral object. Most commercial design packages allow for the export of the mesh object from the CAD model. The common formats for storing mesh files are *.STL and *.obj. Figure 3 shows the difference in various model representations.

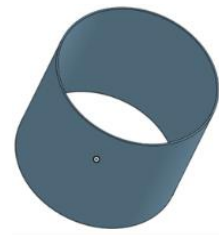

(a)

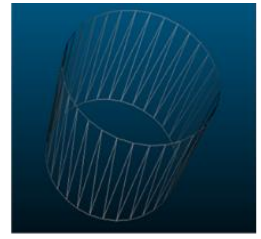

(b)

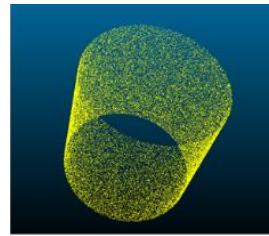

(c)
Figure 3. (a) CAD model representation of a simple cylinder. (b) Mesh representation of the model. (c) Point cloud representation of the model.

To generate a point cloud representation of the model from the input *.STL, the following steps are taken:

1. Parse the STL file, this results in an indexed list of triangles: $1, \ldots, n$

2. Create a new list with n elements, the value of the $i^{\prime}$ th element is the area of the $i$ 'th triangle divided by the sum of the area of all triangles. This is the probability that the i'th triangle will be chosen to sample a point

3. Create a new cumulative probability list by setting the i'th element equal to the sum of the first $i$ element in the previous list

4. Generate a random number from 0 to 1 and find the index of the closest number greater than or equal in the cumulative probability list. This gives an index $j$ of the triangle to sample

5. Pick a random point in the $j$ 'th triangle

6. Repeat steps 4 and 5 for the number of points that you want sampled

\section{Algorithm 2: Generating point representation from} input *.STL

Input: An indexed list of triangles $\left\{t_{1}, t_{2}, \ldots, t_{n}\right\}$ from input *.STL

Output: List of $k$ points $\{P\}$ representing point cloud Create new list $\{A\}$ with $n$ elements

$a_{i}=\frac{\text { Area of } t_{i}}{\sum_{l=1}^{n} \text { Area of } t_{l}}$

Create a cumulative probability list $\{C\}$ with n elements

$c_{i}=\sum_{l=1}^{i} a_{l}$

$P=\{\}$

\section{Repeat $\boldsymbol{k}$ times}

Generate random number $r \in[0,1]$

Find $j$ such that $c_{j}-r \geq 0$ and $c_{j}-r$ is minimized

Append to $\{P\}$ a random point in $t_{j}$

Report $\{P\}$

\subsubsection{Finding the global optimum between scan and model}

This method uses the scan point cloud and the 3D model object as the two inputs. To overlay a scan point cloud on a model point cloud, Principal Component Analysis (PCA) is used for coarse alignment between the two point clouds [27]. Three corresponding points are required as input for this calculation. After obtaining the rough alignment, an Iterative Closest Point (ICP) optimization is implemented to improve the overlay results.

\subsubsection{Finding the local optimum between scan and model}

The next step is to select a region that contains the origin termination points in the scan and model point cloud (classes $A_{s}$ and $A_{m}$ ). This depends on region selection. Once a region is selected, the ICP algorithm is applied to that specific region. The local optimization will result in an improved overlay in the area containing $\left\{A_{m}, \overrightarrow{F_{m}}, A_{s}, \overrightarrow{F_{s}}\right\}$. Figure 4 shows how the region selection is implemented and the final result after updating the overlay.

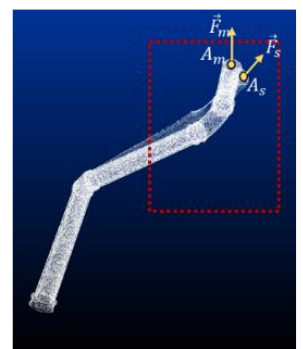

(a)

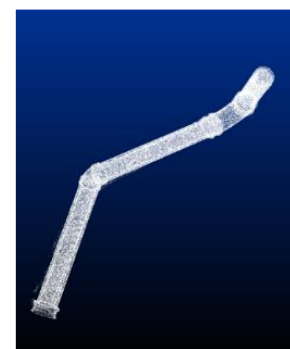

(b)

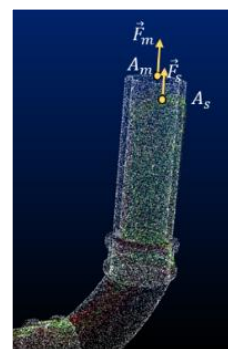

(c)
Figure 4. (a) The overlay after applying the matching step described in Section 3.2.3. The area of interest is selected and outlined by the dotted red square. (b) The result after locally applying ICP to the region of interest. (c) As shown, the local optimization aligns the normal-to-origin termination planes on the 3D model point cloud and as-built point cloud $\left(\overrightarrow{F_{m}} \| \overrightarrow{F_{s}}\right)$.

\subsubsection{Finding the origin termination point on the model point cloud $\left(A_{m}\right)$}

The information of the model's center-point can be theoretically extracted from the CAD file. However, as stated earlier, in many situations, the parametric CAD file is not made available to fabrication shops. As such, the objective in this step is to find the center point on a circular cross-section on the 3D model point cloud (without relying on the original $\mathrm{CAD}$ format) and to capture its coordinate $\left(A_{m}\right)$ in the global coordinate system. The implemented approach requires a user to seed an algorithm that finds $A_{m}$ by selecting a point (via an interactive graphical user interface) on the plane which contains $A_{m}=\left(A_{m x}, A_{m y}, A_{m z}\right)$, and for which the normal vector is parallel with $\vec{F}_{m}=\left(F_{m x}, F_{m y}, F_{m z}\right)$. 
The selection takes place on the mesh object point cloud (where only the vertices are visible to the user); this allows the user to easily visualize the plane containing $A_{m}$. This plane $\left(P_{A_{m}}\right)$ can be defined by Equation (1) as follows:

$$
\begin{gathered}
P_{A_{m}}: \\
F_{m x} X+F_{m y} Y+F_{m z} Z- \\
{\left[F_{m x} A_{m x}+F_{m y} A_{m y}+F_{m z} A_{m z}\right]=0}
\end{gathered}
$$

Once the initial point is selected by the user (on the mesh), an algorithm is developed and described in the following paragraphs to calculate $A_{m}$ and $\vec{F}_{m}$.

Assign $A_{m}=\left(A_{m x}, A_{m y}, A_{m z}\right)$ to be the center point on the 3D model point loud in the global coordinate system. The next step is to structure the vertices on the mesh object in a way that the closest point to an arbitrary point is indexed and searchable. To do this, octree-based data segmentation is used, which allows for the compartmentalization of points into neighbourhoods based on their Euclidean distances. The Octree method divides the 3D space into bins (the bins may or may not be equal in size depending on the method), allows for stopping the subdivision based on the number of points inside a bin (its key advantage in this application over kdtree), and the information regarding the neighbouring bins can also be accessed [28]. Once a point on the plane $P_{A_{m}}$ is selected, two neighbouring points with the shortest Euclidean distance to the selected point are retrieved from the octree data structure. The two calculated points, along with the selected point, are used to define a plane and a circle. Using the three points, a center point, radius, and the normal vector are calculated.

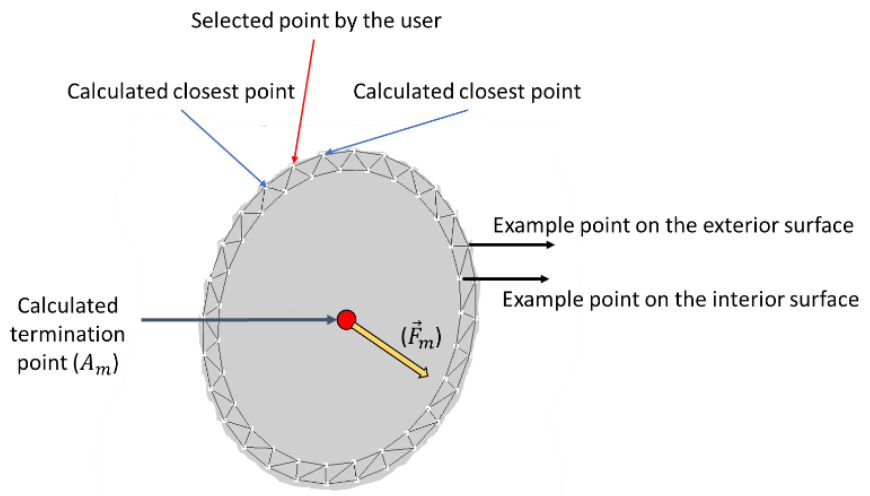

Figure 5. Plane $P_{A_{m}}$ (shown in the mesh representation) is calculated using the mesh object and a user-selected point. The termination point $A_{m}$ and normal-to-origin termination plane $\left(\vec{F}_{m}\right)$ is calculated.

In fabrication projects, the pipe thickness is typically detailed in CAD models, this means that in the mesh object, there will be points corresponding to the exterior surface, and there will be points corresponding to the interior surface of an object (pipe or any other object with circular cross-section). There are cases where even though the user selects a point on the exterior surface, the next closest points lay on the interior surface, or the user inadvertently selects a point on the interior surface. As such, the defined circle by the two closest points to a selected point by the user is incorrect and does not correspond to the center point of the cross-section. In other words, the center of the defined circle does not yield $A_{m}$ due to incorrect points used to construct the circle. To address this issue, an outlier removal method is implemented. As previously described, after selecting a point, the two nearest points to the selected point are calculated. Using the three points, a circle with the termination point as its center and a calculated radius are hypothesized. The hypothesized circle can be described as shown in Equation (2):

$$
C_{A_{m} H}:\left(A_{m H}, R_{A_{m} H}\right)
$$

where $C_{A_{m} H}$ denotes to the hypothesized circle at the origin termination point in the model space, $A_{m H}$ denotes to the hypothesized origin termination point coordinate in the 3D model (hypothesized circle's center), and $R_{A_{m} H}$ denotes the radius of the hypothesized circle. Once the circle is hypothesized, a puck volume is constructed such that the center is $A_{m H}$, the radius is $R_{P u c k}=R_{A_{m} H}+\varepsilon_{1}$, the height of $H_{\text {Puck }}=\varepsilon_{2}$, and where $\varepsilon_{1}$ and $\varepsilon_{2}$ are small values used as padding. Once the puck object is defined, the points encompassed by the volume of the puck are counted. If the number of points captured exceeds the predefined limit, the hypothesized center is accepted as the termination point of that section $A_{m}$. If the number of points is lower than the limit, the next closest point is selected, and all possible circles are constructed. The algorithm will stop when the number of encompassed points exceeds the limit. Figure 6 shows an example where the initial hypothesized circle is incorrect, and the outlier removal algorithm rejects it and finds the correct circle and termination point.

\subsubsection{Finding the origin termination point in scan point $\operatorname{cloud}\left(A_{s}\right)$}

Based on calculations in the previous section, the coordinate of the termination point class $A_{m}\left[A_{m}=\right.$ $\left.\left(A_{m x}, A_{m y}, A_{m z}\right)\right]$, the normal-to-origin termination vector class $\vec{F}_{m}\left[\vec{F}_{m}=\left(F_{m x}, F_{m y}, F_{m z}\right)\right]$, and the radius $R_{A_{m}}$ are known in the global coordinate system. These attributes are used to seed a search; the objective is to detect the termination point class $A_{S}$ along with the radius of the end plane $R_{A_{S}}$ on the as-built point cloud. Note that it is assumed that the normal-to-origin termination point vector classes are parallel between the scan and model. In other words, it is assumed that after applying the local ICP (Section 3.2.4) $\vec{F}_{m} \| \vec{F}_{s}$. The following diagram (Figure7) describes the implementation process for finding the termination point in the scan point cloud $\left(A_{s}\right)$. 


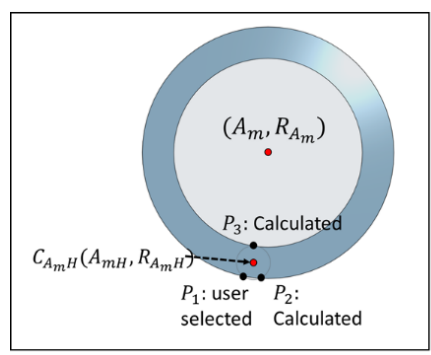

(a)

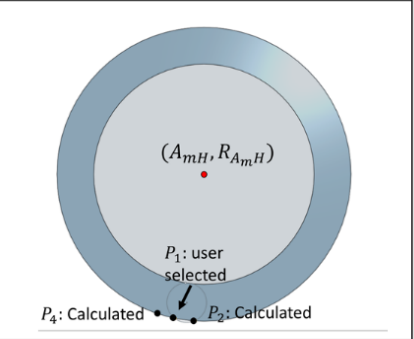

(d)

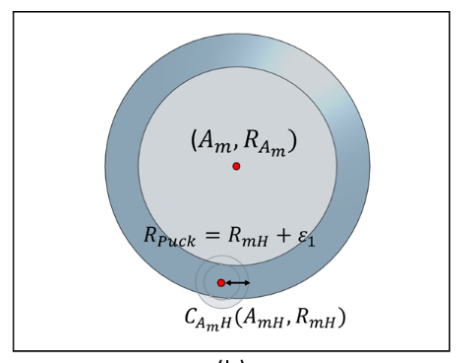

(b)

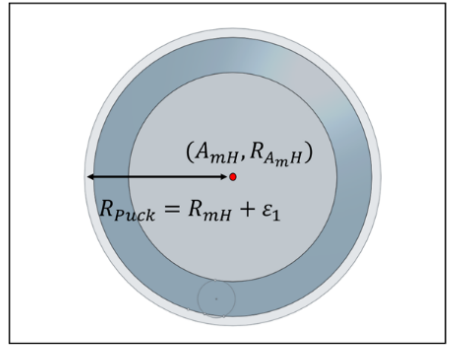

(e)

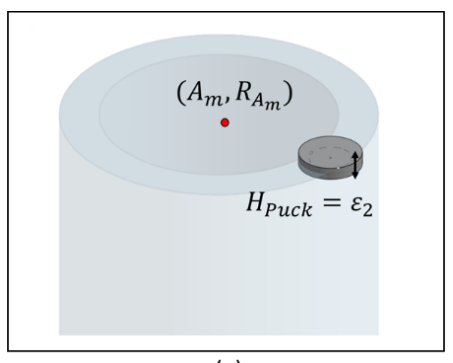

(c)

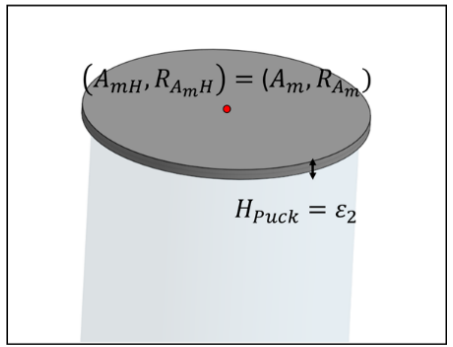

(f)

Figure 6. (a) Top view of the plane $P_{A_{m}} . P_{1}$ selected by the user. Existence of $P_{3}$ in the initial set of closest points results in an erroneous cross-section termination point detection. (b) Creation of the puck volume. (c) The number of points encompassed by the created puck is lower than the predefined limit. (d) The next closest point is selected. (e) Creation of the puck object. (f) The volume encompassed by the puck object exceeds the limit, and the hypothesized circle is accepted.

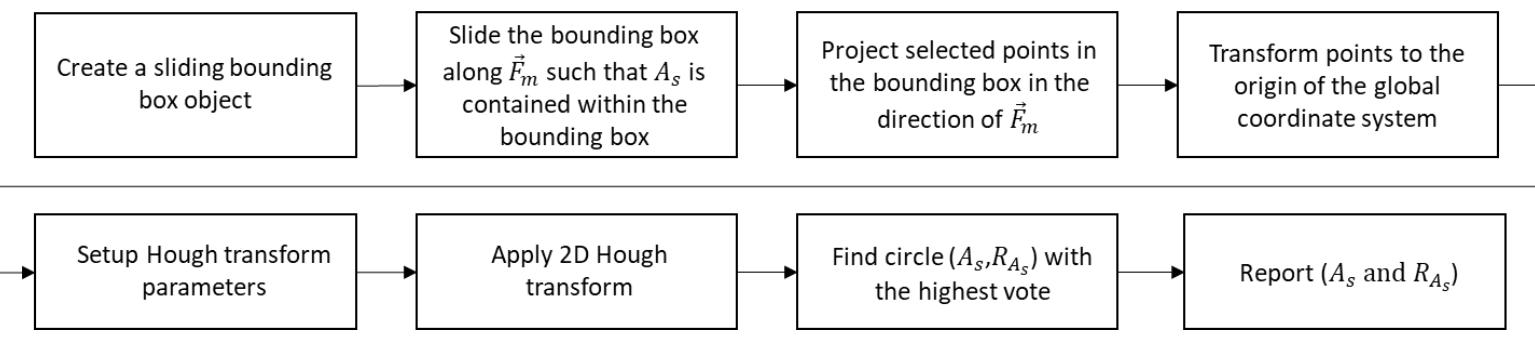

Figure 7. The implementation process for finding the termination point class $A_{S}$ and the radius of the section $R_{A_{S}}$

\subsubsection{Creating the bounding box object}

A bounding box object can be created in the global coordinate system by using a six-element array with elements corresponding to:

$$
\left(x_{\min }, x_{\max }, y_{\min }, y_{\max }, z_{\min }, z_{\max }\right)
$$

Bounding box dimensions $=$

$$
\left\{\begin{array}{c}
-r+\text { increment } \times \text { BoundingBoxIndex } \\
-r+\text { increment } \\
\times(\text { BoundingBoxIndex }+1) \\
-r \times \text { width } \\
r \times \text { width } \\
-r \times \text { height } / 2 \\
r \times \text { height } / 2
\end{array}\right.
$$

The BoundingBoxIndex is a user-controlled value. The BoundingBoxIndex is a method to allow users to select the bounding box that includes the termination point class $A_{s}$ in the scan point cloud.

\subsubsection{Finding the bounding box that contains the} termination point class $A_{s}$

To find the bounding box that contains the termination point $A_{s}$, the defined bounding box needs to be aligned with the calculated $\vec{F}_{m}$. The objective is to align the vector created by [BoundingBoxDimensions[1] BoundingBoxDimensions[0]] with $\vec{F}_{m}$ (the principal axis of the component that contains the termination point $\left.A_{s}\right)$. To align an arbitrary vector $(\vec{u})$ with an arbitrary vector $(\vec{v})$, Rodriguez's rotation formula is used [28]:

$$
\begin{gathered}
\vec{k}=\vec{u} \times \vec{v} \\
K=\left[\begin{array}{ccc}
0 & -k_{y} & k_{y} \\
k_{z} & 0 & -k_{x} \\
-k_{y} & k_{x} & 0
\end{array}\right] \\
\boldsymbol{R}=\boldsymbol{I}+(\sin \theta) \boldsymbol{K}+(1-\cos \theta) \boldsymbol{K}^{2}
\end{gathered}
$$




$$
\boldsymbol{T}=\left[\begin{array}{cccc}
R_{(1,1)} & R_{(1,2)} & R_{(1,3)} & c_{x} \\
R_{(2,1)} & R_{(2,2)} & R_{(2,3)} & c_{y} \\
R_{(3,1)} & R_{(3,2)} & R_{(3,3)} & c_{z} \\
0 & 0 & 0 & 1
\end{array}\right]
$$

$\vec{F}_{m}$ should be substituted in the above equation by $\vec{v}$ to align the bounding box object with the termination plane. Once the rotation has been determined, the bounding box is transformed to the correct location. This is done by applying the rotation matrix that was determined in the previous step and then translating to where the termination point class $A_{m}$ is located (calculated in Section 3.2.4). Figure 8 shows an example illustrating how the bounding box is aligned with $\vec{F}_{m}$ and the contained points by the bounding box are highlighted.

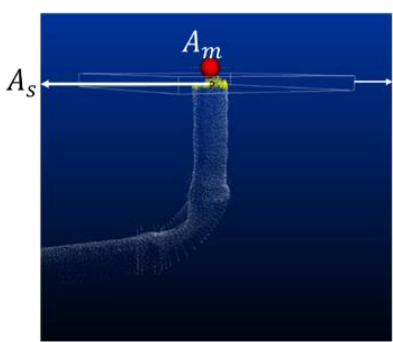

(a)

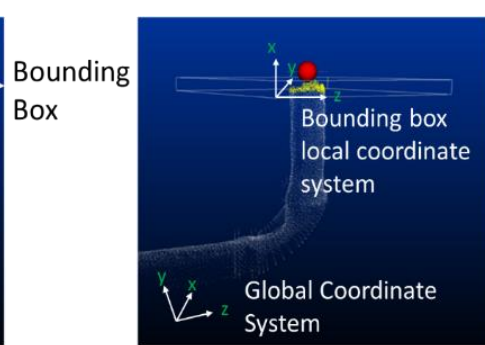

(b)
Figure 8. (a) The bounding box object is created and transformed. $A_{s}$ is included in the bounding box. The points contained in the bounding box object are highlighted. (b) The local coordinate system of the bounding box and the global coordinate system are shown.

\subsubsection{Projecting points within the bounding box onto a plane}

Once the bounding box is created and transformed, the points inside the bounding box can then be selected. The next step is to find the center point using the selected subset of points. The bounding box center point is required when projecting points.

BoundingBoxPoint $_{1}=\left[\begin{array}{l}\text { BoundingBoxDimesions }[0] \\ \text { BoundingBoxDimesions 1] } \\ \text { BoundingBoxDimesions 2] }\end{array}\right]$

BoundingBoxPoint $_{2}=\left[\begin{array}{l}\text { BoundingBoxDimesions [3] } \\ \text { BoundingBoxDimesions [4] } \\ \text { BoundingBoxDimesions [5] }\end{array}\right]$

BoundingBoxCenter $r_{\text {beforeTransform }}=$

$$
\frac{\text { BoundingBoxPoint }_{1}+\text { BoundingBoxPoint }_{22}}{2}
$$

$b b c=$ Bounding Box Center $=$

$$
T * \text { BoundingBoxCenter }_{\text {beforeTransform }}
$$

The coordinates of the selected points (scan point cloud) are described in the global coordinate system. To project along $\vec{F}_{m}$ (the principal axis of the bounding box). The coordinates must be described in the local coordinate system of the Bounding box (Figure 8(b)). Let's name the coordinate of the bounding box center (described in the global coordinate system) as $b b c=\left(b b c_{x}, b b c_{y}, b b c_{z}\right)$. Given that the $\mathrm{R}$ for rotating from the global coordinate to the local coordinate system is calculated (Section 3.2.5), applying the following transformation will transform all selected points within the bounding box to the local coordinate system of the bounding box. Once the selected points are transformed to the origin of the local coordinate system, the points within the bounding box can now be projected onto the plane with $\vec{n}=\vec{F}_{m}$ :

$T_{\text {Global to Local }}=\left[\begin{array}{cccc}R_{(1,1)} & R_{(1,2)} & R_{(1,3)} & b b c_{x} \\ R_{(2,1)} & R_{(2,2)} & R_{(2,3)} & b b c_{y} \\ R_{(3,1)} & R_{(3,2)} & R_{(3,3)} & b b c_{z} \\ 0 & 0 & 0 & 1\end{array}\right]^{-1}$

A column vector is then created, representing each point, and then each point is multiplied by $T_{\text {Global to Local }}$.

Point in Bounding Box Coordinate System $=$

$$
\left[\begin{array}{cccc}
R_{(1,1)} & R_{(1,2)} & R_{(1,3)} & b b c_{x} \\
R_{(2,1)} & R_{(2,2)} & R_{(2,3)} & b b c_{y} \\
R_{(3,1)} & R_{(3,2)} & R_{(3,3)} & b b c_{z} \\
0 & 0 & 0 & 1
\end{array}\right]^{-1} \quad \times\left[\begin{array}{c}
x_{i} \\
y_{i} \\
z_{i} \\
1
\end{array}\right]=\left[\begin{array}{c}
x_{i}^{\prime} \\
y_{i}^{\prime} \\
z_{i}^{\prime} \\
1
\end{array}\right]
$$

After performing the multiplication, the points contained in the bounding box can be described in the bounding box's coordinate system. To project the points within the bounding box, the $\mathrm{x}^{\prime}$ element has to be suppressed ( $\mathrm{X}$ direction of the coordinate system is aligned with $\vec{F}_{m}$ ). The depiction of $y^{\prime}$ and $z^{\prime}$ values for each point in a Cartesian 2D coordinate system will then represent the projection of the points within the bounding box onto the plane $P_{A_{S}}$ with the normal vector equal to $\vec{F}_{S}$. Figure 9 shows a plot of the section in the $\mathrm{z}^{\prime}$ and $\mathrm{y}^{\prime}$ axis.
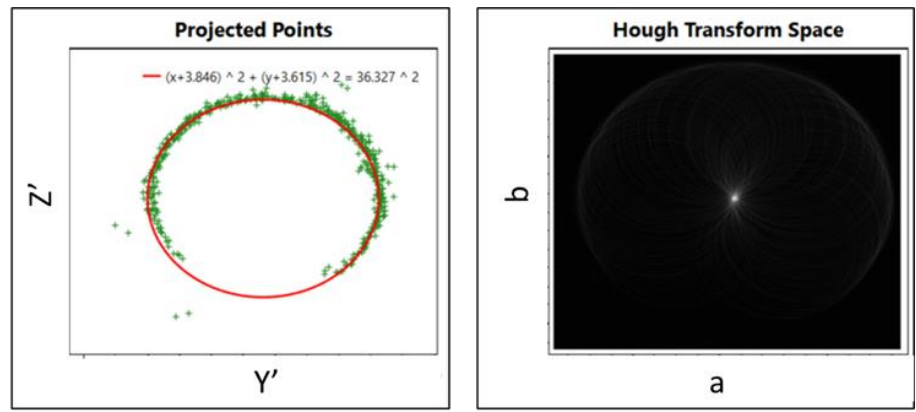

Figure 9. Detection of $A_{s}$ and $D_{A_{s}}$ using Hough transform

3.2.6.4 Circle Hough transform to detect $A_{s}$ and $R_{A_{S}}$ A guided Hough transform is used to best-fit a circle to the projected data points. The circle Hough transform is a voting algorithm that allows for the calculation of the diameter $\left(D_{A_{S}}=2 \times R_{A_{S}}\right)$ and center of a circle $\left(A_{s}\right)[29]$. 
In the implementation of the Hough transform, there are three important parameters that will impact its performance:

1. Radius Guess: The search for finding the highest vote needs a starting point. In the suggested workflow in this research, $R_{A_{m}}$ (calculated in the step described in Section 3.2.4) is used as the initial radius guess.

2. Radius Tolerance: Determines the search space as a multiplier of the radius guess.

3. Radius Resolution: Determines the number of intervals within the search space.

\subsubsection{Final transformation and discrepancy analysis} Once the coordinates of $A_{s}$ and $A_{m}$ are known (in the global coordinate system), the initial overlay between the scan and model point clouds (step described in 4.2.2) can be updated such that $A_{s}$ is overlaid onto $A_{m}$ and the orientation of the end planes between the scan and model are aligned $\left(\vec{F}_{m} \| \vec{F}_{s}\right)$. This process can be best described as a digital $3 \mathrm{D}$ jig, where one end is locked and the deviation on the other end is calculated. Once the overlay between the scan and model is updated, a discrepancy analysis can be done between the two data sets outlining the out-of-alignment areas. This can be done by calculating the deviation of each point in the scan point cloud to the closest point on the model point cloud. The results of the discrepancy analysis can be shown in the form of a colour map where points exceeding the tolerance threshold are coloured in red and points below the tolerance threshold are coloured in green. Figure 10 summarizes the steps explained in Section 3.2.

\section{Results}

To validate the accuracy of calculated termination points by the described method, the results of the method were compared against the reports provided by a third-party quality inspection firm. For the inspection of incoming pipe spools, a hand-held laser scanner arm device (FARO Arm; $0.05 \mathrm{~mm}$ accuracy at $100 \mathrm{~mm}$ distance [29]) was used. The fabricator then provided access to the verification reports to the research team, where the diameter at the termination points on the as-built objects was calculated and reported. Since this method is regarded as the industrially accepted method for measuring termination points and their corresponding diameter, these values are used as ground truth. A snapshot of one such report is provided in Figure 11. Additionally, the results of the FARO Arm were crosschecked by using a calibrated calliper. The validation of the FARO Arm results is described in Section 4.1.

Note that the allowable tolerance for termination points in this project was $1.5 \mathrm{~mm}$. The allowable tolerance value is always dictated by the owner's requirement and it is agreed upon before a fabrication job is quoted. In most piping projects, the tolerance dictated by PFI-ES-03 is used as a baseline, and if it is not mentioned otherwise, it is assumed to be the tolerance requirements for an owner.

The dataset collected in this research was also used to find the termination points using a commercial scan-to-BIM software package. The accuracy results of the commercial package were then compared with the developed method (both were benchmarked against the FARO Arm) in Section 4.3.

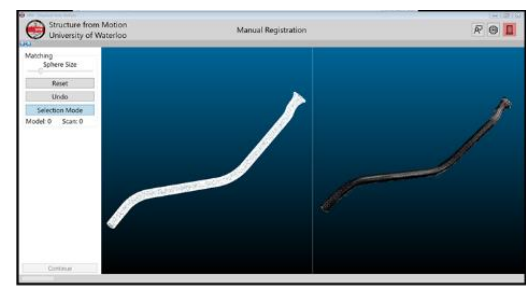

(a)

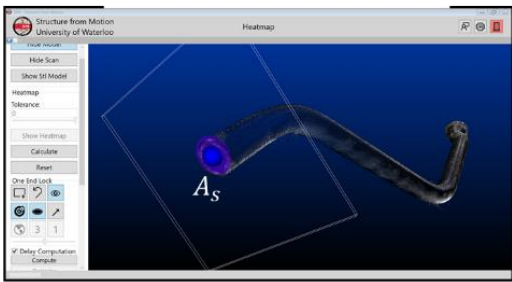

(d)

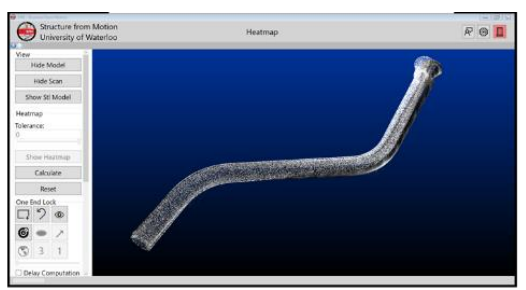

(b)

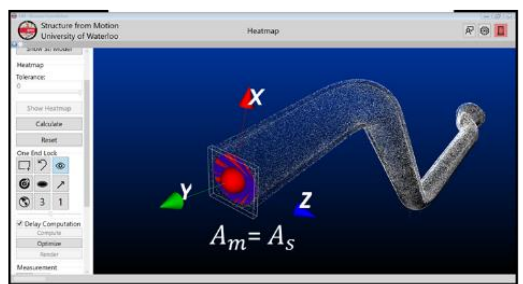

(e)

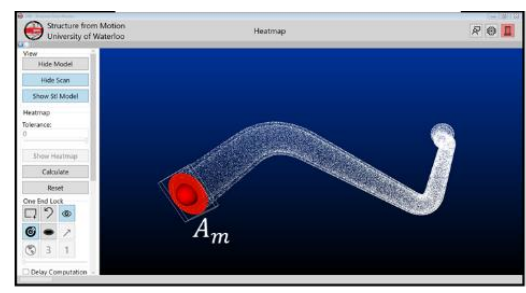

(c)

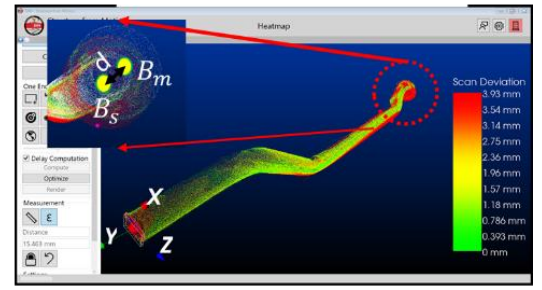

(f)

Figure 10. (a) The model (as-designed) point cloud next to the scanned (as-built) point cloud of a pipe spool. (b) The initial positioning (PCA+ICP, Section 3.2.2). (c) The model origin termination point is detected (Section 3.2.4). (d) The scan origin termination point is calculated (Section 3.2.5). (e) the scan point cloud is transposed such that the scan origin termination point and plane is overlaid on (fitted to) the model origin termination point and plane (Section 3.2.6). (f) The colormap is generated, and the deviation at the destination termination points between model and scan is calculated (Section 3.2.6) 


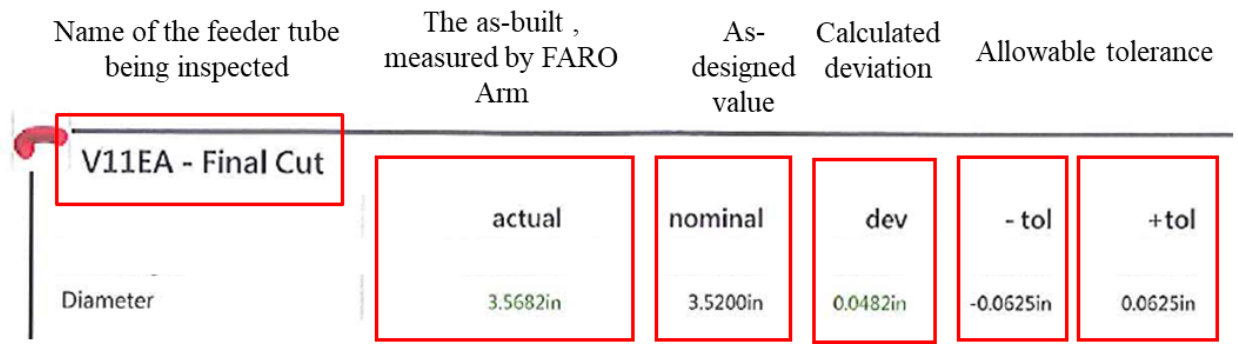

Figure 11. Verification report provided by the third-party company. The allowable tolerance is $1.58 \mathrm{~mm}$. All feeder tubes have a verification report. The calculated deviation is based on measuring the diameter of the feeder tube using the FARO Arm and comparing it with the model.

\subsection{FARO Arm Verification}

To verify that the data provided by the FARO Arm scanner (which the fabricator had hired a surveying crew to measure with) is accurate, a calibrated manual calliper was used to cross-check the provided data.

On average, the difference (between calliper and FARO Arm) in the measured diameters was $0.07 \mathrm{~mm}$, with the maximum difference being $0.39 \mathrm{~mm}$. Since the maximum difference between the calliper readings and the scanner arm was less than the required tolerance on this project $(1.58 \mathrm{~mm}$ or $1 / 16$ '), the readings of the arm scanner were accepted as the ground truth (baseline) for the comparison and benchmarking of the developed method. The absolute point-by-point difference between the calliper's readings and the FARO Arm's readings is illustrated in Figure 12.
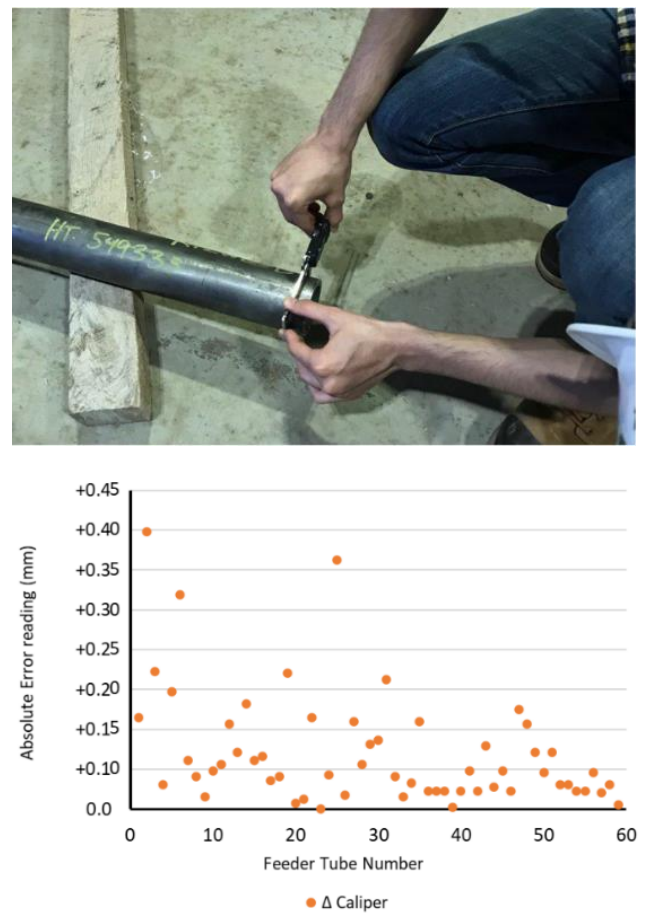

Figure 12. The difference between calliper readings and the FARO Arm

\subsection{Accuracy in Calculation of Model and Scan Origin Termination Points $\left[A_{s}\right.$ and $\left.A_{m}\right]$}

To estimate the accuracy of the calculated coordinates for the origin termination points in the scan and model $\left(A_{s}\right.$ and $\left.A_{m}\right)$, the calculated diameter at the plane in which these points are included is used $\left(D_{A_{m}}\right.$ is the calculated diameter at the plane containing $A_{m}$ and $D_{A_{S}}$ is the calculated diameter at the plane containing $A_{s}$ ). The comparison of diameters will provide a reasonable estimation since both methods (developed method and measuring with the scanner arm) rely on circle fitting algorithms using points captured on the perimeter of the cross-section. Hence, inaccurate diameter calculation will result in inaccurate coordinate estimation, and accurate diameter calculation is conducive to an accurate coordinate calculation for the termination points.

\subsubsection{Diameter Accuracy at the Model Origin Termination Plane $\left(D_{A_{m}}\right)$}

As explained in Section 3.2.1, a polygon mesh format is used for model representation, which uses triangles to represent objects. The process of converting CAD models into a mesh object may cause inaccuracies, which is a function of the settings to generate the mesh file. Additionally, rounding error is inevitable in the process of unit conversation (the models were designed in inches, and all calculations were done in $\mathrm{mm}$ ). Finally, the developed method may also cause some minor errors in estimating the model termination point. As such, the calculated diameter for the model $\left(D_{A_{m}}\right)$ is compared with its nominal $\mathrm{CAD}$ value for all feeder tubes, and the error is reported in Figure 13.

\subsubsection{Diameter Accuracy at the Scan Origin Termination Plane $\left(D_{A_{s}}\right)$ Using Laser Scanner Data Input}

The point clouds of 30 feeder tubes (each with two termination points) and a total of 60 termination points were captured and analyzed. After the initial acquisition by the laser scanner, the point clouds were registered together to create a complete point cloud representation of the as-built objects (multiple scans were acquired and 
stitched together). The $D_{A_{S}}$ was then calculated using the method described in Section 3.2.6. As explained earlier, to determine the $D_{A_{S}}$, the Hough Transform requires three input parameters. The initial radius guess is the calculated $D_{A_{m}}$ (Section 3.2.6.4) since the allowable tolerance for this project was $1.5 \mathrm{~mm}$. The minimum radius tolerance was determined such that the search space would never get smaller than the allowable tolerance. As such, the radius tolerance was set to $2 \%$ (radius tolerance is a proportional metric). This means that, for the smallest test case with a diameter of $70 \mathrm{~mm}$, the search space would be $1.4 \mathrm{~mm}$. To see the impact of radius tolerance and radius resolution, nine value pairs were tested for all captured data to evaluate which value pair provides the best estimation for calculating $D_{A_{S}}$. It should be noted that both the radius tolerance and radius resolution are unitless parameters. For example, when the value pair is set to [radius tolerance $=10 \%$ and radius resolution $=200$ ] for a feeder tube with a nominal diameter of $100 \mathrm{~mm}$, to estimate $D_{A_{S}}$, the algorithm will start with the initial radius guess of $100 \mathrm{~mm}$ and continues its search with steps equal to $\frac{100 \times 0.1}{200} \mathrm{~mm}$ (radius resolution). In this example, the boundary of the neighbourhood in which the search is conducted would be $100 \mathrm{~mm} \pm(100 \times 0.1) \mathrm{mm}$ (radius resolution). Table 3 shows different value pair combinations for performance evaluation.

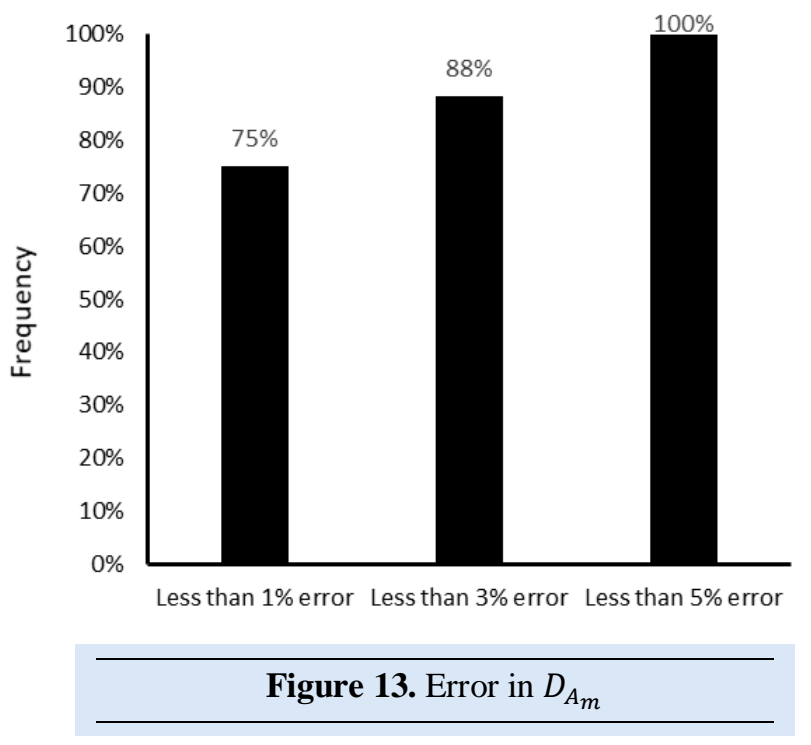

Table 3. Different parameters tested in the developed framework

\begin{tabular}{cccccccccc} 
Parameter & \multicolumn{10}{c}{ Value pairs } \\
\hline $\begin{array}{c}\text { Radius } \\
\text { Resolution } \\
\begin{array}{c}\text { Radius } \\
\text { Tolerance }\end{array}\end{array}$ & 50 & 50 & 50 & 100 & 100 & 100 & 200 & 200 & 200 \\
\hline
\end{tabular}

As shown in Figure 14, the best performance for the laser scanned data with an average absolute error of $1.01 \mathrm{~mm}$ (average relative error as a percent is equal to $1.18 \%$ ) and standard deviation of 0.62 was obtained when the radius resolution was set to $2 \%$ and the radius tolerance was set to 200 . Additionally, when set to $[2 \%, 200]$ value pair, the maximum error as an absolute value was $2.76 \mathrm{~mm}$, the maximum error as a percent was $3.05 \%$, the absolute minimum error was $0.09 \mathrm{~mm}$, and the minimum error as a percent was $0.2 \%$.

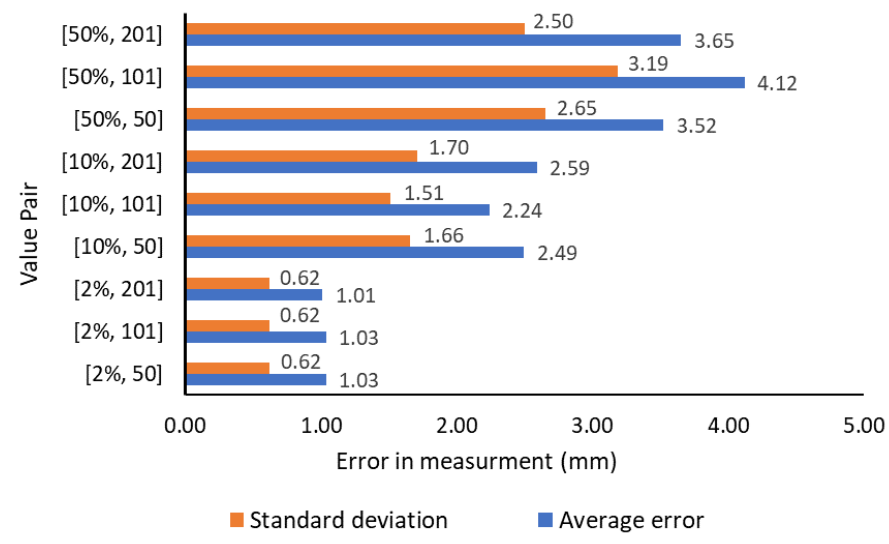

Figure 14. Evaluation of best value pair for Hough Transform based on laser scanned data input

Finally, using the optimized value pair, the frequencyerror calculations for all termination points are shown in Figure 15.

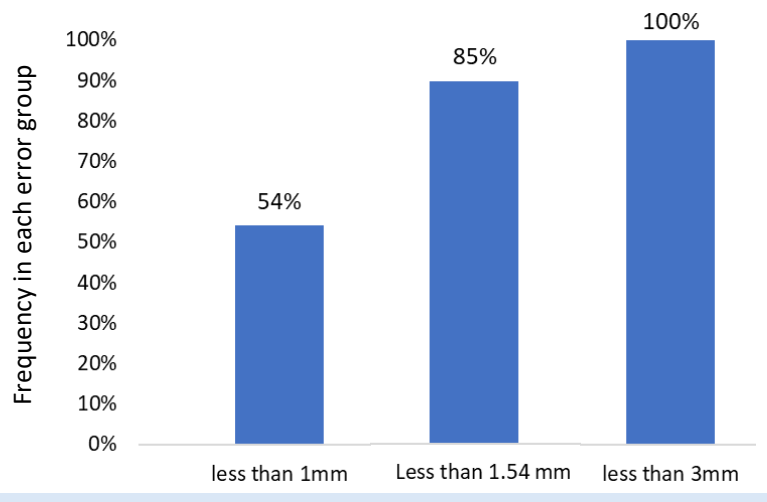

Figure 15. The frequency of error based on absolute error

\subsection{Comparison with Commercial Scan-to-BIM}

In addition to testing the method with different parameters, the obtained point clouds were used to measure the accuracy of utilizing a commercial software package for converting the scan point clouds to BIM objects. In this study, Edgewise ${ }^{\circledR}$ was used to convert the obtained as-built point clouds to BIM objects. The diameter of the feeder tube was then extracted as an attribute of the BIM object. The extracted diameter was compared against the ground truth, and the performance was compared against the developed method. 
Using the laser scanner data combined with the developed method provided improved accuracy in $91 \%$ of the cases. The developed method has an average error of $1.01 \mathrm{~mm}$, a standard deviation of 0.62 , a maximum error of 2.76 $\mathrm{mm}$, and a minimum error of $0.09 \mathrm{~mm}$. Comparatively, the Edgewise software solution has an average error of $4.01 \mathrm{~mm}$, a standard deviation of 4.75 , a maximum error of $22.3 \mathrm{~mm}$, and the minimum error being $0.02 \mathrm{~mm}$. The results are further illustrated in Table 4 and Figure 16.

In $15 \%$ of the cases, the error of the developed method is more than the required accuracy. This is due to (1) the used laser scanner, and (2) the used method for registration of the scans. The laser scanner used in this study is a lower-tier scanner with an average accuracy of $3 \mathrm{~mm}$; as such, since the input data in the method doesn't necessarily have the required accuracy, the developed method fails to completely compensate. Additionally, due to the fast pace of fabrication, in this study, two laser scanners were used at the same time and targetless registration methods were used. The utilization of these methods while facilitating faster scanning time, can add additional inaccuracies for the input data. Finally, while the developed method does not have sufficient accuracy in $15 \%$ of the cases, it has sufficient accuracy for all the cases under the requirements by PFI-ES-03. This is important, since most piping projects follow the requirements suggested by this standard. While the algorithm described may be complex, its use as a function called by an interactive user interface can be made quite simple, intuitive and appropriate for an untrained technician or for a pipe fitter or QC person.

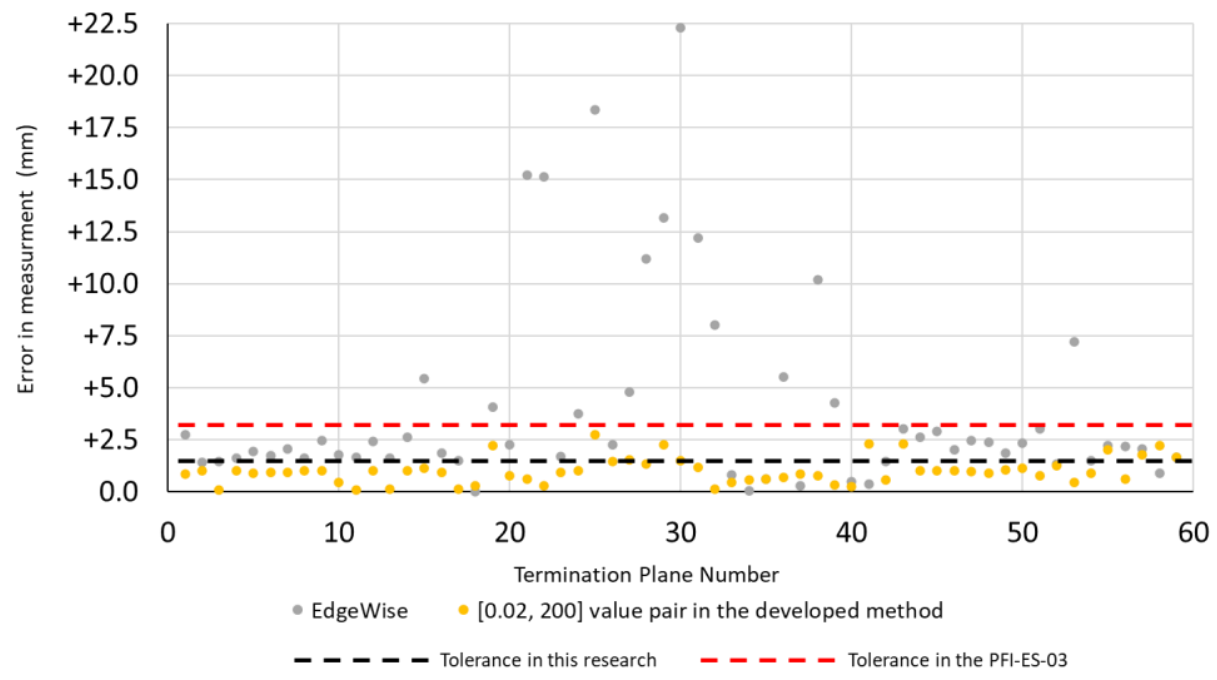

Figure 16. The measurement error in using both methods has been shown with a laser scanner as their data input source. The reference measurement is data collected by the probing unit.

Table 4. Performance comparison results with the laser scanner as the data input source

\begin{tabular}{lcc}
\hline Method & Edgewise & Developed method \\
\hline Average error $(\mathrm{mm})$ & +4.01 & 1.01 \\
Standard deviation & 4.75 & 0.62 \\
Maximum error $(\mathrm{mm})$ & 22.3 & 2.76 \\
Minimum error $(\mathrm{mm})$ & 0.02 & 0.09 \\
\hline
\end{tabular}

\section{Conclusions and Recommendations}

The recent growth in prefabrication and modular projects requires fabrication shops to re-evaluate their existing measurement and quality control processes. Existing measurement processes rely heavily on manual hand measurement tools, which often yield deficient accuracy. Aware of uncertainties with manual measurement tool-based methods and with the increased complexity of assemblies, project owners and designers require more frequent QC steps to reduce the risk of dimensional issues, which in turn causes increased project completion time, increased rework cost, and fabrication worker frustration. To solve this problem and equip fabrication workers with an advanced measurement tool, 3D measurement systems can potentially be deployed. In the present study, a method for accurate detection and measurement of termination points was provided. 
To determine the accuracy of detecting termination points in the scan and model point clouds, the calculated diameter in the circle fitting algorithm was used as an approximation. The final registered point cloud was used for the measurement of point cloud accuracy. This is important, since the hardware equipment can only certify an average accuracy in each scan and does not account for errors in the registration step. In the developed method, the calculated diameter in the model space is used to seed a search and to approximate the diameter in the scan space. Thus, the developed method provided an improved accuracy using the laser scanner compared to the method described by Nahangi et al. [24].

The developed method in this research was used in conjunction with traditional quality control tools for the fabrication of feeder tubes. As part of this research project, the method detected 7 non-compliant pipe spools, which was later confirmed by the QC team upon further investigation.

It should be noted that while only $85 \%$ of detected termination points had an accuracy below the required tolerance in this project $(1.54 \mathrm{~mm})$, all termination points had an accuracy below $3 \mathrm{~mm}$. The $3 \mathrm{~mm}$ threshold is important since most projects follow the tolerance requirements recommended by PFI-ES-03 [30] which has a $3 \mathrm{~mm}$ tolerance recommendation on most angles and distances.

Laser scanners also proved to be a reliable and accurate source of $3 \mathrm{D}$ data. While their utilization is less mobile and more time-consuming compared to SLAM scanners, their use may be required based on the required accuracy. Furthermore, to reduce the time required with a laser scanner, in this paper, multiple laser scanners were used at the same. Additionally, existing commercial software can be used to streamline and automate the data acquisition and point cloud registration aspect of laser scanners.

In future work, data communication across different stakeholders can be improved using Augmented Reality. Creating correspondence between a colour map deviation point cloud and the fabricated assembly may be cumbersome on large symmetrical shapes. Superimposing deviation maps onto built assemblies can potentially save time in data interpretation and communication. Additionally, 3D models can be projected onto assemblies that are being built as an indication for the next steps and guiding welders and fitters as they are building assemblies. However, improved productivity by better communication and real-time guidance through Augmented Reality has to be extensively researched and investigated.

\section{Acknowledgments}

The authors would like to thank Scott Waters, Chas Williams, and Stacey Jenson Rose from their industrial partner, Aecon Group, who supported this research through a Collaborative Research and Development (CRD) grant with the Natural Sciences and Engineering Research Council (NSERC) Canada. The authors would also like to thank Steve Chuo, Jackie Bai, Abdullah Majid, and Minren Hung for their efforts in data collection and supporting this research. This research is partially funded by Mitacs E-Accelerate and Glove Systems Inc.

\section{References}

[1] Noguchi, M. (2003). The effect of the quality-oriented production approach on the delivery of prefabricated homes in Japan. Journal of Housing and the Built Environment, 18, 353-364. https://doi.org/10.1023/B:JOHO.0000005759.07212.00

[2] Tchidi, M.F., He, Z., \& Li, Y.B. (2012). Process and quality improvement using Six Sigma in construction industry. Journal of Civil Engineering and Management, $18(2)$, 158-172. https://doi.org/10.3846/13923730.2012.657411

[3] Kwiatek, C., Sharif, M., Li, S., Haas, C., \& Walbridge, S. (2019). Impact of augmented reality and spatial cognition on assembly in construction. Automation in Construction, 108 , 102935 .

https://doi.org/10.1016/j.autcon.2019.102935

[4] Irizarry, J., Gheisari, M., \& Walker, B.N. (2012). Usability assessment of drone technology as safety inspection tools. Journal of Information Technology in Construction (ITcon), 17, 194-212.

[5] McKinsey \& Company. (2020). The next normal in construction. https://www.mckinsey.com/businessfunctions/operations/our-insights/the-next-normal-inconstruction-how-disruption-is-reshaping-the-worldslargest-ecosystem

[6] Yap, J.B.H., Low, P.L., \& Wang, C. (2017). Rework in Malaysian building construction: Impacts, causes and potential solutions. Journal of Engineering, Design and Technology, 15(5), 591-618. https://doi.org/10.1108/JEDT-01-2017-0002

[7] Love, P. E. D., Edwards, D., Watson, H., \& Davis, P. (2010). Rework in civil infrastructure projects: Determination of cost predictors. Journal of Construction Engineering and Management, 136(3), 275-282. https://doi.org/10.1061/(ASCE)CO.1943-7862.0000136

[8] Chuo, S. (2020). Simulation modelling and analysis of impact of $3 d$ feedback workflow on prefabrication of industrial construction. UWSpace. http://hdl.handle.net/10012/16280 
[9] Goodrum, P.M., Miller, J., Sweany, J., \& Alruwaythi, O. (2016). Influence of the format of engineering information \& spatial cognition on craft-worker performance. Journal of Construction Engineering \& Management, 142(9), 04016043. https://doi.org/10.1061/(ASCE)CO.19437862.0001157

[10] Bae, J., \& Han, S. (2021). Vision-based inspection approach using a projector-camera system for off-site quality control in modular construction: Experimental investigation on operational conditions. Journal of Computing in Civil Engineering, 35(5), 04021012. https://doi.org/10.1061/(ASCE)CP.1943-5487.0000978

[11] Safa, M., Shahi, A., Nahangi, M., Haas, C., \& Noori, H. (2015). Automating measurement process to improve quality management for piping fabrication, Structures, 3, 71-80. https://doi.org/10.1016/J.ISTRUC.2015.03.003

[12] Guo, J., Wang, Q., \& Park, J. (2020). Geometric quality inspection of prefabricated MEP modules with 3D laser scanning. Automation in Construction, 111, 103053. https://doi.org/10.1016/j.autcon.2019.103053

[13] Ahmed, M.F., Haas, C.T., \& Haas, R. (2014). Automatic detection of cylindrical objects in built facilities. Journal of Computing in Civil Engineering, 28(3), 4014009. https://doi.org/10.1061/(ASCE)CP.1943-5487.0000329

[14] Son, H., Kim, C., \& Kim, C. (2015). Fully automated asbuilt 3D pipeline extraction method from laser-scanned data based on curvature computation. Journal of Computing in Civil Engineering, 29(4), B4014003. https://doi.org/10.1061/(ASCE)CP.1943-5487.0000401

[15] Wang, B., Yin, C., Luo, H., Cheng, J.C.P., \& Wang, Q. (2021). Fully automated generation of parametric BIM for MEP scenes based on terrestrial laser scanning data. Automation in Construction, 125, 103615. https://doi.org/10.1016/j.autcon.2021.103615

[16] Dimitrov, A., Gu, R., \& Golparvar-Fard, M. (2016). Nonuniform B-spline surface fitting from unordered 3D point clouds for as-built modeling. Computer-Aided Civil and Infrastructure Engineering, 31(7), 483-498. https://doi.org/10.1111/mice.12192

[17] Maalek, R., Lichti, D.D., Walker, R., Bhavnani, A., \& Ruwanpura, J.Y. (2019). Extraction of pipes and flanges from point clouds for automated verification of prefabricated modules in oil and gas refinery projects. Automation in Construction, 103, 150-167. https://doi.org/10.1016/j.autcon.2019.03.013

[18] Esfahani, M.E., Rausch, C., Sharif, M.M., Chen, Q., Haas, C., \& Adey, B.T. (2021). Quantitative investigation on the accuracy and precision of Scan-to-BIM under different modelling scenarios. Automation in Construction, 126, 103686. https://doi.org/10.1016/j.autcon.2021.103686

[19] Nguyen, C.H.P., \& Choi, Y. (2018). Comparison of point cloud data \& 3D CAD data for on-site dimensional inspection of industrial plant piping systems. Automation
in
Construction,
91
$44-52$.
https://doi.org/10.1016/j.autcon.2018.03.008

[20] Guo, J., Wang, Q., \& Park, J. (2020). Geometric quality inspection of prefabricated MEP modules with 3D laser scanning. Automation in Construction, 111, 103053. https://doi.org/10.1016/j.autcon.2019.103053

[21] Nahangi, M., \& Haas, C.T. (2016). Skeleton-based discrepancy feedback for automated realignment of industrial assemblies. Automation in Construction, 61, 147-161. https://doi.org/10.1016/j.autcon.2015.10.014

[22] Nahangi, M., Yeung, J., Haas, C.T., Walbridge, S., \& West, J. (2015). Automated assembly discrepancy feedback using 3D imaging and forward kinematics. Automation in Construction, 56, 36-46. https://doi.org/10.1016/j.autcon.2015.04.005

[23] Kalasapudi, V.S., Tang, P., \& Turkan, Y. (2017). Computationally efficient change analysis of piece-wise cylindrical building elements for proactive project control. Automation in Construction, 81, 300-312. https://doi.org/10.1016/j.autcon.2017.04.001

[24] Nahangi, M., Czerniawski, T., Haas, C.T., \& Walbridge, S. (2019). Pipe radius estimation using Kinect range cameras. Automation in Construction, 99, 197-205. https://doi.org/10.1016/j.autcon.2018.12.015

[25] Abu-Nabah, B.A., ElSoussi, A.O., \& Al Alami, A.E.K. (2018). Virtual laser vision sensor environment assessment for surface profiling applications. Measurement, 113, 148160. https://doi.org/10.1016/j.measurement.2017.08.052

[26] Sharif, M.M., Haas, C., \& Walbridge, S. (2021). Using termination points and $3 \mathrm{D}$ visualization for dimensional control in prefabrication. [Manuscript submitted for publication]. Department of Civil and Environmental Engineering, University of Waterloo.

[27] Wold, S., Esbensen, K., \& Geladi, P. (1987). Principal component analysis. Chemometrics and Intelligent Laboratory Systems, 2(1-3), 37-52. https://doi.org/10.1016/0169-7439(87)80084-9

[28] Carlbom, I., Chakravarty, I., \& Vanderschel, D. (1985). A hierarchical data structure for representing the spatial decomposition of 3-D objects. IEEE Computer Graphics and Applications, 5(4), 24-31. https://doi.org/10.1109/MCG.1985.276454

[29] FARO. (2017). Accuracy Specifications for the Gage Arm. https://knowledge.faro.com/Hardware/Legacy-

Hardware/Legacy-

Gage/Accuracy_Specifications_for_the_Legacy_Gage_F aroArm (Accessed date October 2, 2021).

[30] Pipe Fabrication Institute Engineering Committee. (2000). Fabricating tolerances. Pipe Fabrication Institute. https://fdocuments.net/document/pfi-es-03-2000.html (Accessed date October 2, 2021). 